\title{
Evaluation of side effects of radiofrequency capacitive hyperthermia with magnetite on the blood vessel walls of tumor metastatic lesion surrounding the abdominal large vessels: an agar phantom study
}

\author{
Vascular Cell 6:15 ～DOI: 10.1186/2045-824X-6-15 ｜ＣＬi et al.; licensee Publiverse Online S.R.L. 2014 \\ Received: 23 Feb 2014 I Accepted: 8 Feb 2014 | Published: 15 Feb 2014 \\ Kawai Noriyasu@ ${ }^{@}$, Kobayashi Daichi, Yasui Takahiro, Umemoto Yukihiro, Mizuno Kentaro, Okada Atsushi, \\ Tozawa Keiichi, Kobayashi Takeshi, Kohri Kenjiro \\ ${ }^{+}$Contributed equally ${ }^{@}$ Corresponding author
}

\begin{abstract}
Background

Magnetite used in an 8-MHz radiofrequency (RF) capacitive heating device can increase the temperature of a specific site up to $45^{\circ} \mathrm{C}$. When treating a metastatic lesion around large abdominal vessels via hyperthermia with magnetite, heatinginduced adverse effects on these vessels need to be considered. Therefore, this study examined hyperthermia-induced damage to blood vessel walls in vitro.
\end{abstract}

\section{Methods}

A large agar phantom with a circulatory system consisting of a swine artery and vein connected to a peristaltic pump was prepared. The blood vessels were placed on the magnetite-containing agar piece. Heating was continued for 30 min at $45^{\circ} \mathrm{C}$. After heating, a histological study for injury to the blood vessels was performed.

\section{Results}

The inner membrane temperature did not reach $45^{\circ} \mathrm{C}$ due to the cooling effect of the blood flow. In the heated vessels, vascular wall collagen degenerated and smooth muscle cells were narrowed; however, no serious changes were noted in the vascular endothelial cells or vascular wall elastic fibers. The heated vessel wall was not severely damaged; this was attributed to cooling by the blood flow.

\section{Conclusions}

Our findings indicate that RF capacitive heating therapy with magnetite may be used for metastatic lesions without injuring the surrounding large abdominal vessels.

\section{Keywords}

Magnetic cationic liposome - Interstitial hyperthermia injury - Large vessels - Vascular cell damage - Agar phantom study

\section{Introduction}

Mammalian cell death due to fever was described by Coss et al. [ 1], who demonstrated that Chinese hamster ovary (CHO) cells continued to survive owing to a colony-forming capacity despite warming at increasing temperatures for a constant time. It was learned that at temperatures less than $42.5^{\circ} \mathrm{C}$, the cell survival rate does not decrease rapidly, but at $43^{\circ} \mathrm{C}$ and above survival decreases linearly. Thus, $42.5^{\circ} \mathrm{C}$ is a critical temperature for cell death occurring due to fever, and hyperthermia emerged as a promising cancer therapy. As a result, various hyperthermia treatments have been attempted [ 2 , 3]. However, an unavoidable technical problem remains, i.e., even localized heating of cancer tissue at a specific temperature induces damage in the surrounding, normal tissues. In contrast, capacitive heating of tumors using a radiofrequency $(\mathrm{RF})$ electric field is a method of hyperthermia that is commonly employed in actual clinical practice in Japan. However, capacitive heating is unsuitable for site-specific hyperthermia because currently available methods do not allow specific heating of the tumors alone. 
In capacitive hyperthermia the normal tissue is also warmed in the part picked up with an electrode. The specific adsorption rate of electric energy depends on the electrical properties, such as permittivity and electric resistance of each tissue type. Since there are no significant differences in the electrical properties of tumor and normal tissues, it is difficult to specifically heat only the tumor. Therefore, in order to prevent excessive heating of normal tissue, "mild" heating conditions of less than $40^{\circ} \mathrm{C}$. are often applied. With this method, heating of the tumor is often insufficient, and complete suppression of the tumor is rather difficult. To overcome this problem, Kobayashi et al. developed magnetite cationic liposomes (MCLs) as fine magnetic particles of submicron size for use in inductive hyperthermia [ 4, 5]. MCLs were devised in order to improve adsorption by tumor cells and accumulation efficiency; these particles have a 10-times higher affinity for tumor cells than fine magnetic particles with no electric charge [ 4]. Many studies have reported the effectiveness of hyperthermia using MCLs in vivo [ 4,6]. We have also previously performed in vivo studies on the application of hyperthermia using MCLs for prostate cancer therapy [ 7,8] and have reported in vivo findings concerning its therapeutic effects on recurrent prostate cancer and bone metastases [9]. Kobayashi et al. demonstrated that magnetic nanoparticles serve as a medium that induces efficient heat generation in RF-capacitive heating [10]. In an in vivo experiment, they observed that heat generation using magnetic nanoparticles was similar to that using inductive heating employing an alternating magnetic field. Thus, with mild hyperthermia that is tolerable for patients, it is possible to generate heat greater than $42.5^{\circ} \mathrm{C}$ with $\mathrm{MCL}$ injection, thereby reaching the critical temperature for cancer cell death. In contrast, other tissues that have not been injected with MCL do not experience an increase in temperature beyond $42.5^{\circ} \mathrm{C}$ even if picked up with an electrode. Therefore, magnetic nanoparticles are considered to be a promising heat-generating medium not only for inductive heating but also for capacitive hyperthermia [11].

For lymph node metastases and local recurrence of urinary system cancers that are located deep within the trunk, tumorspecific hyperthermia using MCLs with RF-capacitive heating appears to be a possible treatment modality. However, such lesions are typically adjacent to the abdominal aorta, inferior vena cava, or the common, internal, or external iliac artery/vein. When capacitive heating is applied after the injection of MCLs into such targets, the temperature of MCLinjected lesions may reach $42.5^{\circ} \mathrm{C}$ as well as the surrounding tissues. If adjacent great blood vessels are injured by this heat, then massive life-threatening bleeding would occur. Therefore, before performing such RF-capacitive hyperthermia using MCLs for lymph node metastases and/or local recurrences of urothelial cancers, it is necessary to confirm that this therapy does not cause injury to the great vessels near the tumor tissue.In this study, we prepared a model consisting of swine blood vessels and an agar phantom for simulating deep trunk lymph node treatment with capacitive heating by MCL injection, and investigated the influence of hyperthermia treatment on the blood vessels.

\section{Materials and methods}

\section{Preparation of MCL}

MCLs were fabricated from colloidal magnetite (a kind gift from Toda Kogyo, Hiroshima, Japan) and a lipid mixture consisting of N-(a-trimethylammonioacetyl)- didodecyl-D-glutamate chloride (Sogo Pharmaceutical Co., Ltd., Tokyo, Japan), dilauroylphosphatidylcholine, and dioleoylphosphatidylethanolamine (Sigma-Aldrich, St. Louis, MO) in a molar ratio of 1:2:2, as described previously [3].

\section{Swine blood vessels}

Based on studies performed by research institutions following animal study guidelines, the abdominal aorta and vena cava were excised from a 3-month-old female pig being euthanized, and the cranial and caudal sides were cut to prepare vascular tubes of approximately $20 \mathrm{~cm}$ in length. Small branches radiating out from the arterial wall were excised, and the openings were closed by ligature/suture and application of an adhesive. Plastic connectors (Connecter 456; Tokyo Garasu Kikai Co., Ltd., Tokyo) were attached to both ends. One end was connected to a peristaltic pump (SJ-1211H; ATTO Co., Ltd., Tokyo), while the other opened into an effluent bucket, thereby preparing a liquid circuit in the blood vessel. This preparation was approved by the appropriate ethics review boards.

\section{Preparation of agar phantom}

A 4\% agar gel was used for preparation of the phantom, using agar purchased from Wako Pure Chemicals Co., Ltd., Tokyo. A small cylindrical agar piece containing the magnetite (magnetite piece) was prepared by a modification of the method described by Jordan et al [ 12]. In brief, the magnetite was added to liquid agar at $60^{\circ} \mathrm{C}$, while stirring with a glass impeller. After mixing for $30 \mathrm{~min}$, the suspension was sonicated for $15 \mathrm{~min}$ at $20 \mathrm{~W}$ using a probe-type sonicator. The sonicated mixture $(20 \mathrm{~mL})$ was poured into a polypropylene cylinder $(28 \mathrm{~mm}$ in diameter) and then rapidly cooled by placing the cylinder in ice water. Figure $1 \mathrm{~A}$ shows a schema of the phantom. Two holes were made along the centerline in the large phantom piece $(300 \times 200 \times 100 \mathrm{~mm})$, and the cylindrical magnetite piece was inserted into the piece. A groove was then made on the inserted, cylindrical magnetite piece in order to place a blood vessel in parallel with the y-axis. The blood vessel was placed in contact with the cylindrical magnetite piece. The remaining space was filled with $4 \%$ agar. An agar piece without magnetite for control experiments (control piece) was also inserted in a symmetric position with respect to the central axis of the electrode.

\section{Figure 1}




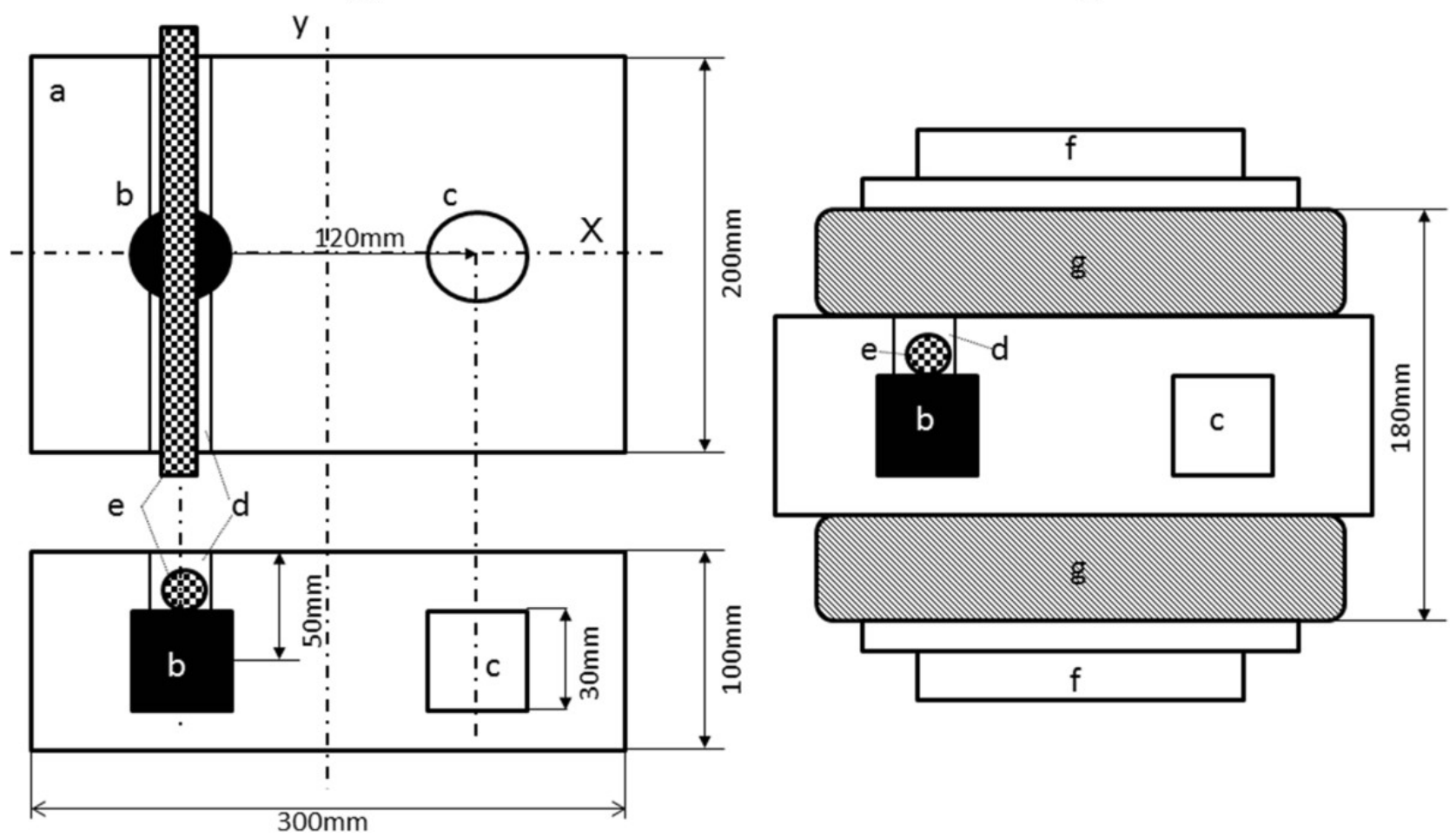

Experimental setup.Schemas of the agar phantom (A)and with the electrode setting (B)a: Agar phantom, b: cylindrical magnetite piece, c: control piece without magnetite, d: groove prepared for setting a blood vessel, e: vessel, f: electrode, g: water cooling pad The cylindrical magnetite and control pieces were symmetrically placed against the y-axis on the central axis of the agar phantom. A groove was made parallel to the y-axis, and a blood vessel was placed in contact with the cylindrical magnetite piece.

\section{In vitro $\mathrm{RF}$ hyperthermia}

An 8-MHz RF capacitive heating device (Thermotron RF-8; Yamamoto Vinyter Co., Osaka) was used. The diameter of the pair of electrodes used for the phantom was $25 \mathrm{~cm}$. The electrodes were covered with a water pad, and cooling water was circulated at $5^{\circ} \mathrm{C}$. The configuration of the phantom and electrodes is shown in Figure $1 \mathrm{~B}$. Power control of the electrodes was performed by the standard method used in routine clinical situations by the Department of Urology, Nagoya City University Medical School.

The temperature was measured using a Teflon-coated microthermocouple that was inserted into the phantom through a 21gauge angiocatheter. The microthermocouples were connected to the Thermotron RF-8 or a recorder (Yokogawa Electric Co., Ltd., Tokyo).The microthermocouples were set at 2 sites: immediately beside the blood vessel located directly above the cylindrical magnetite piece, and directly above the control piece. The flow speed was set at $14 \mathrm{~mL} / \mathrm{s}$, which was the fastest rate of the peristaltic pump for both the artery and vein. Heating was continued for $30 \mathrm{~min}$ after the temperature directly above the cylindrical magnetite piece reached $45^{\circ} \mathrm{C}$. Further, 30-min heating was applied once while saline was perfused in the artery or the vein and to another vein without saline perfusion.After the heating was completed, the blood vessel was removed from the agar. The regions that were in contact with the cylindrical magnetite piece and adequate sections from the distant ends were excised and subjected to histological examination.

\section{Immunohistochemical staining and special histochemical analysis}

Vascular damage was evaluated by staining elastic fibers, collagen, vascular endothelium, and smooth muscle employing Elastica van Gieson (EVG) [ 13], Sirius Red [ 14], von Willebrand factor (vWF) [ 15], and $\alpha$-smooth muscle actin (SMA) staining [16], respectively.

Immunohistochemical staining was applied to $3 \mu \mathrm{m}$ thick paraffin sections of the swine artery and vein. Sections were incubated for $60 \mathrm{~min}$ with antibodies specific to SMA (Dako Cytomation Inc.) and Factor VIII-related antigen (Dako Cytomation Inc.). Staining was achieved using the 2-step EnVision + System-HRP methodology, according to the manufacturer's instructions (Dako Cytomation Inc.). Sections were lightly counterstained with hematoxylin to facilitate orientation. Immunostained slides were evaluated by light microscopy, and the proportion of positively stained cells (positivity) was scored from the tumor, normal tissue, and the nuclear and cell membranes.

\section{Results}

\section{Heating}

Figure 2 shows the schema for heating of the agar phantom and blood vessel using RF-8. The container shown in (k) of Figure 2 A contained physiological saline, and saline was pumped up through the silicon tube (i) connected to the 
peristaltic pump (h). Saline flowed into the blood vessel (e), passing through the agar phantom (a), and discharged into the silicon tube (j). Probes measured the temperature of the region directly adjoining the blood vessel (b) and the control piece (c). Figure 2B shows temperature measurements observed during heating using thermography (Thermography R300; NEC/Avio Co., Ltd., Tokyo). As shown by the scale on the right, red and blue colors represent $45^{\circ} \mathrm{C}$ and $8^{\circ} \mathrm{C}$, respectively. Since $5^{\circ} \mathrm{C}$ cooling water was circulated in the water pad while heating, thermography displayed the color blue representing a low temperature. The color red, representing $45^{\circ} \mathrm{C}$, is displayed beside the blood vessel (b') in contact with the cylindrical magnetite piece in the agar phantom, whereas the color green, representing $\sim 30^{\circ} \mathrm{C}$, is observed for the control piece (c'). The inflow silicon tube (i) was colored blue-green (i'), showing that the inflow saline temperature was approximately $25^{\circ} \mathrm{C}$. The outflow silicon tube (j), into which saline flowing out of the blood vessel passed, was colored yellow (j'), showing that the outflowing saline was heated to $\sim 35^{\circ} \mathrm{C}$. Figure $2 \mathrm{C}$ shows the time-course temperatures in the blood vessel (b) and control piece (c) from the point of initiation of heating using RF-8. In the blood vessel, the temperature reached $45^{\circ} \mathrm{C}$ at $28 \mathrm{~min}$ after heating initiation, while in the control piece, the temperature reached $37^{\circ} \mathrm{C}$ after 37 min, but no further increase in temperature was observed. Heating was continued for $30 \mathrm{~min}$ after the temperature reached $45^{\circ} \mathrm{C}$ in the blood vessel and for $30 \mathrm{~min}$ after it reached $37^{\circ} \mathrm{C}$ in the control piece.

\section{Figure 2}

A

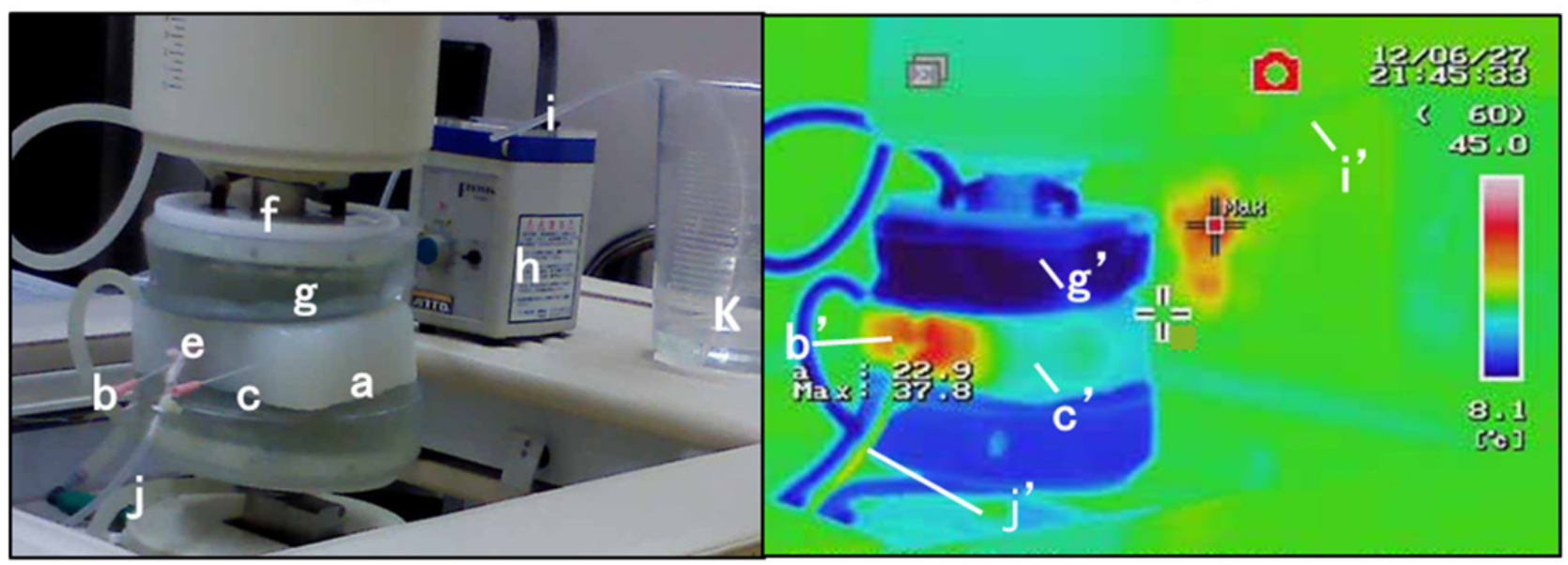

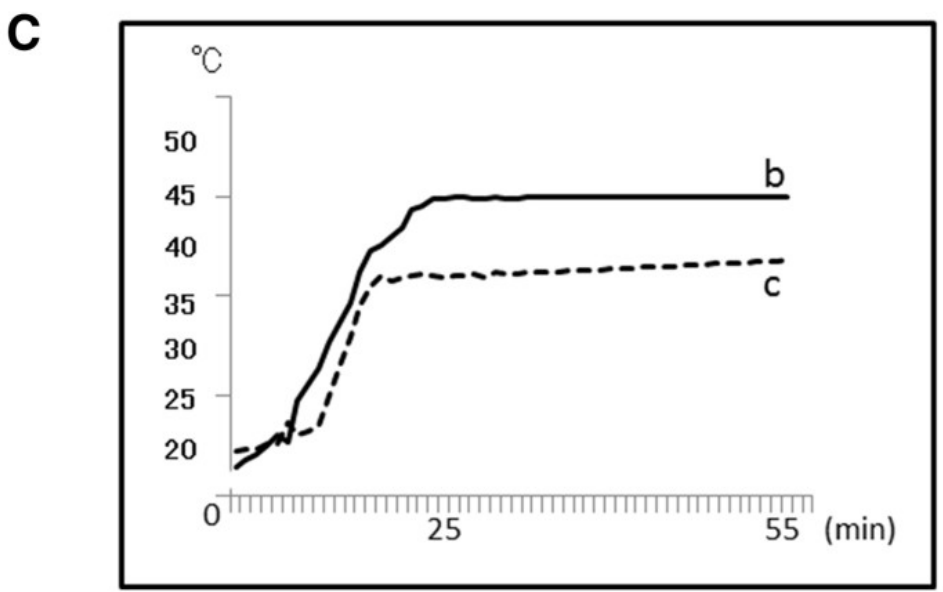

Heating. (A)Explanation of RF-8. a-g: same as Figure 1. h: peristaltic pump, i: silicon tube for inflow, j: silicon tube for outflow, k: saline. (B)Thermography. b': Temperature display of the cylindrical magnetite piece, approximately $45^{\circ} \mathrm{C}$. c': Temperature display of the control piece, approximately $30^{\circ} \mathrm{C}$. g': Temperature display of the water cooler pad, approximately $8^{\circ} \mathrm{C}$. j': Temperature display of the silicon tube for outflow, approximately $35^{\circ} \mathrm{C}$. i’: Temperature display of the silicon tube for inflow, approximately $20^{\circ} \mathrm{C}$. (C)Time course of temperature changes in b and c. b: The temperature reached $45^{\circ} \mathrm{C}$ at $27 \mathrm{~min}$ after the initiation of heating and remained at this temperature thereafter. c: The temperature reached $35^{\circ} \mathrm{C}$ at $23 \mathrm{~min}$ and did not exceed $37^{\circ} \mathrm{C}$ thereafter.

With the power of the RF-generator turned on, the temperature of the control piece reaches $42^{\circ} \mathrm{C}$. In clinical situations the control piece would represent the normal tissue packed by the electrodes of RF-8, so at $42^{\circ} \mathrm{C}$. the control piece is at an intolerable temperature for the patient. Thus our hyperthermia method using MCL and Thermotron RF- 8 was activated when the temperature of the magnetite-containing agar piece, which was in contact with the target organ reached $45^{\circ} \mathrm{C}$., while the control piece mimicking normal tissue is kept under $37^{\circ} \mathrm{C}$. This hyperthermia assembly was shown to be useful for the treatment of deeply positioned lymph node metastases.

\section{Change in various tissues of vascular wall}

\section{Discussion}


The aim of the present study was to determine the effects of heating tumors located in deep regions around great blood vessels up to temperatures of $45^{\circ} \mathrm{C}$ by using histological and immunohistochemical examinations. Chen et al [ 17] previously reported a mathematical model, in which hyperthermia was simulated to coagulate tumors by inserting electrodes of an RF-ablation device, wherein heat dissemination to the surrounding region was measured, as well as determining the distance limit not affecting the adjacent great blood vessels. The influence of high heat on great vessels can be avoided in hyperthermia by using an RF-ablation device since electrodes can be freely inserted. However, the thermal effect of such devices extends only to several millimeters from the electrode, which precludes the treatment of lesions greater than $2 \mathrm{~cm}$.

In our proposed hyperthermia procedure, MCLs would be injected into tumors followed by external RF irradiation. In addition to RF-induced biological heat generation, the tumor would be heated internally to temperatures up to $45^{\circ} \mathrm{C}$ by injecting MCLs that serve as a heating medium. These MCLs would necrotize the tumor by heating from the center, slowly expanding the region to the tumor periphery, suggesting that the temperature in the tumor periphery also rises to nearly $45^{\circ} \mathrm{C}$ as treatment continues [ 10,11$]$. Saline circulation was included for simulating blood flow in order to accurately evaluate injuries to the great vessels adjacent to tumors heated to $45^{\circ} \mathrm{C}$.

In clinical stage, metastatic lymph nodes targeted by our proposed hyperthermia often exist around large vessels. Thus, these adjacent vessels may also be exposed to high temperatures approaching $45^{\circ} \mathrm{C}$ during such hyperthermic treatment of tumors. This technique differs from the hyperthermia induced by using an RF-ablation device. Therefore, the histological evaluation of vascular damage following exposure to high temperature, as performed in this study, was necessary.

Our results confirmed that the cylindrical MCL agar was heated up to $45^{\circ} \mathrm{C}$ using capacitive hyperthermia, as reported by Kobayashi et al. [ 11]. Heating to temperatures above $50^{\circ} \mathrm{C}$ was possible when the output of the RF generator RF-8 was increased, and the temperature in the control piece could be increased to $45^{\circ} \mathrm{C}$ (data not shown).

Regarding vascular wall impairment, the thin venous wall was assumed to suffer more severe damage from heat treatment than the strong arterial wall. Thus, a model of heating without saline perfusion of the vein was used, which was believed would further elevate the temperature because of the absence of a heat-releasing mechanism. Subsequently, immunohistochemical staining was performed to examine elastic fibers, collagen, and smooth muscle in the artery and veins to evaluate vascular damage. This included the vascular wall structures and vascular endothelium, which were included because they were exposed to perfusion within the vascular lumen. Elastic fibers were unchanged by heating, a finding common to the artery and the vein, while collagen was markedly degenerated following heating. Further, ruptured and defective regions were present in the vein that was not perfused with saline, in which the temperature may have risen to nearly $45^{\circ} \mathrm{C}$. The number of smooth muscle cells was not reduced in either the arterial or venous wall, although individual cells became smaller.Regarding the temperature in the vascular lumen, the saline outflow was about $30^{\circ} \mathrm{C}$ on thermography, suggesting that the vascular lumen, i.e., the vascular endothelial cells, were cooled to about $35^{\circ} \mathrm{C}$. The lumen was also flattened in the heated artery due to elastic fiber deformity, but no changes were noted in the vascular endothelial cells. Moreover, in the vein without perfusion, no loss of endothelial cells was noted; however, the cells were narrowed. This cellular narrowing occurred to a lesser degree in the vein heated with perfusion than in the vein without perfusion.

This study used an isolated artery and vein, in which injured tissues did not recover. However, such tissues may regenerate in vivo, and collagen and smooth muscle may regenerate in time. Therefore, although the collagen and smooth muscle experienced some amount of degeneration, since the vascular endothelium was not ruptured and the elastic fibers were not damaged, it is conceivable that the tube structure would be maintained and fatal complications may not occur in such localized RF-capacitive hyperthermia therapy.

We submit that our hyperthermia method using MCL and Thermotron RF-8 is safe for treating deeply positioned metastatic lymph nodes.

Our present findings are expected to be useful as the preliminary data required for such an in vivo study.

\section{Conclusions}

The present study indicates that RF-capacitive heating therapy with magnetite may be used for metastatic lesions that surround large abdominal vessels, without injuring these vessels.

\section{Ethical approval}

Institutional Animal Care and Use Committee.

\section{Declarations}

\section{Acknowledgments}

We thank Takahiro Teraoka (Nippon Avionics Co., Ltd.) who provided thermography equipment for temperature evaluation. Further, we thank Naomi Kasuga, Rina Kawamura, Kyoko Ichikawa, and Momoko Noda, engineer (Department of Nephro-urology, Nagoya City University Graduate School of Medical Sciences) for their technical assistance and cooperation. 


\section{Authors' original submitted files for images}

Below are the links to the authors' original submitted files for images.

Authors' original file for figure 1

Authors' original file for figure 2

Authors' original file for figure 3

Authors' original file for figure 4

\section{Competing interests}

The authors declared that they have no competing interests.

\section{Authors' contributions}

NK, DK, TY, KM carried out this hyperthermic experiment. NK, AO and KT carried out the histochemical examination. TK and KK advised and supported this experiment from the basic and clinical stand points. NK drafted the manuscript. All authors read and approved the final manuscript.

\section{References}

1. Coss RA, Messinger JA, Wahl ML, Wachsberger PR, Leeper DB, Owen CS. Bicarbonate-dependent proton extrusion in Chinese hamster ovary $(\mathrm{CHO})$ cells adapted to growth at $\mathrm{pH}$ 6.7. Int J Hyperthermia. 1997; 13:325-336.

View Article Google Scholar

2. Overgaard K, Overgaard J. Investigation on the possibility of a thermic tumour therapy. II. Action of combined heat-roentgen treatment on a transplanted mouse mammary carcinoma. Eur J Cancer. 1972;8:573575.

$\underline{\text { View Article Google Scholar }}$

3. Lyons BE, Britt RH, Strohbehn JW. Localized hyperthermia in the treatment of malignant brain tumors using an interstitial microwave antenna array. IEEE Trans Biomed Eng. 1984;31:53-62.

$\underline{\text { View Article Google Scholar }}$

4. Shinkai M, Yanase M, Honda H, Wakabayashi T, Yoshida J, Kobayashi T. Intracellular hyperthermia for cancer using magnetite cationic liposomes: in vitro study. Jpn J Cancer Res. 1996;87:1179-1183.

View Article Google Scholar

5. Yanase M, Shinkai M, Honda H, Wakabayashi T, Yoshida J, Kobayashi T. Intracellular hyperthermia for cancer using magnetite cationic liposomes: an in vivo study. Jpn J Cancer Res. 1998;89:463-469.

View Article Google Scholar

6. Yanase M, Shinkai M, Honda H, Wakabayashi T, Yoshida J, Kobayashi T. Intracellular hyperthermia for cancer using magnetite cationic liposomes: ex vivo study. Jpn J Cancer Res. 1997;88:630-632.

View Article Google Scholar

7. Kawai N, Ito A, Nakahara Y, Futakuchi M, Shirai T, Honda H, Kobayashi T, Kohri K. Anticancer effect of hyperthermia on prostate cancer mediated by magnetite cationic liposomes and immune-response induction in transplanted syngeneic rats. Prostate. 2005;64:373-381.

View Article Google Scholar

8. Kawai N, Ito A, Nakahara Y, Honda H, Kobayashi T, Futakuchi M, Shirai T, Tozawa K, Kohri K. Complete regression of experimental prostate cancer in nude mice by repeated hyperthermia using magnetite cationic liposomes and a newly developed solenoid containing a ferrite core. Prostate. 2006;66:718-727.

View Article Google Scholar

9. Kawai N, Futakuchi M, Yoshida T, Ito A, Sato S, Naiki T, Honda H, Shirai T, Kohri K. Effect of heat therapy using magnetic nanoparticles conjugated with cationic liposomes on prostate tumor in bone. Prostate. 2008;68:784-792.

View Article Google Scholar

10. Shinkai M, Ueda K, Ohtsu S, Honda H, Kohri K, Kobayashi T. Effect of functional magnetic particles on radiofrequency capacitive heating. Jpn J Cancer Res. 1999;90:699-704. 
11. Shinkai M, Ueda K, Ohtsu S, Honda H, Kohri K, Kobayashi T. Effect of functional magnetic particles on radiofrequency capacitive heating: an in vivo study. Jpn J Cancer Res. 2002;93:103-108.

View Article Google Scholar

12. Jordan A, Wust P, Fähling H, John W, Hinz A, Felix R. Inductive heating of ferrimagnetic particles and magnetic fluids: physical evaluation of their potential for hyperthermia. Int J Hyperthermia. 1993;9:51-68. View Article Google Scholar

13. Kepes JJ, Yarde WL. Visualization of injected embolic material (polyvinyl alcohol) in paraffin sections with Verhoeff-Van Gieson elastica stain. Am J Surg Pathol. 1995;19:709-711.

View Article Google Scholar

14. Travers JP, Brookes CE, Evans J, Baker DM, Kent C, Makin GS, Mayhew TM. Assessment of wall structure and composition of varicose veins with reference to collagen, elastin and smooth muscle content. Eur J Vasc Endovasc Surg. 1996;11:230-237.

View Article Google Scholar

15. Christensen S, Verhage HG, Nowak G, de Lanerolle P, Fleming S, Bell SC, Fazleabas AT, Hild-Petito S. Smooth muscle myosin II and alpha smooth muscle actin expression in the baboon (Papio anubis) uterus is associated with glandular secretory activity and stromal cell transformation. Biol Reprod. 1995;53:598-608. View Article Google Scholar

16. Mustafa S, Thulesius O, Elgazzar AH, Ismael HN. Synergistic effects of ethanol and hyperthermia on carotid artery vasoconstriction. Clin Physiol Funct Imaging. 2007;27:185-190.

View Article Google Scholar

17. Chen X, Saidel GM. Mathematical modeling of thermal ablation in tissue surrounding a large vessel. J Biomech Eng. 2009;131:011001-.

View Article Google Scholar 